\title{
Activation of Nrf2 by Sulforaphane Inhibits High Glucose-Induced Progression of Pancreatic Cancer via AMPK Dependent Signaling
}

\author{
Xin Chen ${ }^{\mathrm{a}}$ Zhengdong Jiang ${ }^{\mathrm{a}}$ Cancan Zhou ${ }^{\mathrm{a}} \quad \mathrm{Ke}^{\mathrm{C}}$ Chen ${ }^{\mathrm{a}} \quad$ Xuqi Li ${ }^{\mathrm{b}} \quad$ Zheng Wang $^{\mathrm{a}}$ \\ Zheng $\mathrm{Wu}^{\mathrm{a}}$ Jiguang $\mathrm{Ma}^{\mathrm{c}}$ Qingyong Ma ${ }^{\mathrm{a}}$ Wanxing Duan ${ }^{\mathrm{a}}$ \\ aDepartment of Hepatobiliary Surgery, First Affiliated Hospital, Xi'an Jiaotong University, Xi'an, \\ ${ }^{b}$ Department of General Surgery, First Affiliated Hospital, Xi'an Jiaotong University, Xi'an, 'Department \\ of Anesthesiology, First Affiliated Hospital, Xi'an Jiaotong University, Xi'an, China
}

\section{Key Words}

Sulforaphane $\cdot$ High glucose $\cdot$ Pancreatic cancer $・$ Nrf2-AMPK signaling

\begin{abstract}
Background/Aims: Sulforaphane (SFN) is known for its potent bioactive properties, such as anti-inflammatory and anti-tumor effects. However, its anti-tumor effect on pancreatic cancer is still poorly understood. In the present study, we explored the therapeutic potential of SFN for pancreatic cancer and disclosed the underlying mechanism. Methods: Panc-1 and MiaPaca-2 cell lines were used in vitro. The biological function of SFN in pancreatic cancer was measured using EdU staining, colony formation, apoptosis, migration and invasion assays. Reactive oxygen species (ROS) production was measured using 2'-7'-Dichlorofluorescein diacetate (DCF-DA) fluorometric analysis. Western blotting and immunofluorescence were used to measure the protein levels of p-AMPK and epithelial-mesenchymal transition (EMT) pathway-related proteins, and cellular translocation of nuclear factor erythroid 2-related factor 2 (Nrf2). Nude mice and transgenic pancreatic cancer mouse model were used to measure the therapeutic potential of SFN on pancreatic cancer. Results: SFN can inhibit pancreatic cancer cell growth, promote apoptosis, curb colony formation and temper the migratory and invasion ability of pancreatic cancer cells. Mechanistically, excessive ROS production induced by SFN activated AMPK signaling and promoted the translocation of Nrf2, resulting in cell viability inhibition of pancreatic cancer. Pretreatment with compound $\mathrm{C}$, a small molecular inhibitor of AMPK signaling, reversed the subcellular translocation of Nrf2 and rescued cell invasion ability. With nude mice and pancreatic cancer transgenic mouse, we identified SFN could inhibit tumor progression, with smaller tumor size and slower tumor progression in SFN treatment group. Conclusion: Our study not only elucidates the mechanism of SFN-induced inhibition of pancreatic cancer in both normal and high glucose condition, but also testifies




\section{Cellular Physiology Cell Physiol Biochem 2018;50:1201-1215

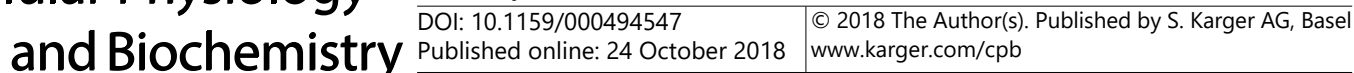 \\ Chen et al.: Sulforaphane Inhibits Pancreatic Cancer}

the dual-role of ROS in pancreatic cancer progression. Collectively, our research suggests that SFN may serve as a potential therapeutic choice for pancreatic cancer.

\section{Introduction}

Pancreatic cancer is a lethal tumor characterized by early metastasis and a high recurrence rate, with a dismal 5-year survival rate of less than 8\% [1-3]. Unlike many other cancers, patients remain almost symptomless in the early stage of this disease. Despite the great progress has been made in the last decades, surgical intervention remains the optimal treatment option. However, once diagnosed, patients with pancreatic cancer usually lose the opportunity to undergo radical surgery. Chemotherapy, a common therapy for these patients with late-stage pancreatic cancer, usually has little effect as a result of serious side effects and low sensitivity. With the increasing incidence of pancreatic cancer, it will be considered the leading cause of cancer-related deaths in America by 2050 [4]. Therefore, identifying new drugs or therapeutic treatments to address this troublesome disease is urgently needed.

Epidemiologic studies indicate that hyperglycemia is an important risk factor for pancreatic cancer [5, 6]. Additionally, about $45-60 \%$ of patients with pancreatic cancer suffer from diabetes [7]. Patients using antidiabetics is associated with reduced pancreatic cancer risk $[8,9]$. Recent studies conducted by our team have revealed that hyperglycemia can not only promote cell migration and proliferation but also potentiate the perineural invasion of pancreatic cancer, a new path for cancer metastasis. In addition, chronic hyperglycemia can exacerbate the desmoplastic reaction through elevating the expression of CXCL12, FOS, and LTBP2, which will then activate pancreatic stellate cells [10].

Sulforaphane (SFN, 4-methylsufinyl-3-butenyl isothiocyanate), an isothiocyanate, is known for its potent role in cancer therapy. SFN is widely found in cruciferous vegetables, such as broccoli, Chinese kale, turnips and cabbage. Previous studies have indicated that SFN has many biological properties, including anti-oxidant, anti-inflammatory, and anti-bacterial effects $[11,12]$. SFN has been found to act as a tumor suppressor in many different cancers during cancer progression, including oral, breast, liver, colon, and prostate cancers [13-16]. In addition, it has been demonstrated that SFN has the ability to prevent carcinogen-induced initiation of oral cancer [14]. Inducing apoptosis and cell cycle arrest are the main factors attributed to the anti-cancer capabilities of SFN. Recently, researchers revealed that SFN can also promote the immune response in a mouse model of leukemia through enhancing the activities of macrophages and natural killer cells [17]. In pancreatic cancer, SFN can downregulate the proliferation and angiogenesis of pancreatic stem cells by inhibiting the sonic hedgehog signaling pathway $[18,19]$. SFN can inhibit pancreatic tumour-initiating

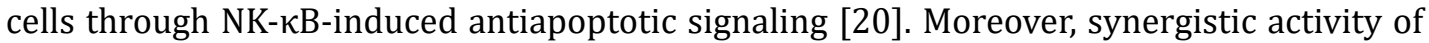
sorafenib and SFN may be an effective choice for targeting pancreatic cancer stem cells [21]. Currently, little is known about the biological role of SFN in high glucose-induced promotion of pancreatic cancer.

In this article, we aim to explore the role of SFN in the biological progression of pancreatic cancer under normal glucose or high glucose conditions. We not only demonstrate the unfavorable role of SFN in pancreatic cancer cells cultured in normal glucose medium but also show that SFN can impair tumor progression under high glucose conditions. The AMPK signaling pathway, an important pathway in cell metabolism, is confirmed to be essential in this process. Inhibition of AMPK phosphorylation by a small molecular inhibitor dramatically reduces the anti-cancer effect of SFN. 


\section{Cellular Physiology Cell Physiol Biochem 2018;50:1201-1215 \begin{tabular}{ll|l} 
and Biochemistry & $\begin{array}{l}\text { DOI: 10.1159/000494547 } \\
\text { Published online: 24 October } 2018\end{array}$ & $\begin{array}{l}\text { (c) } 2018 \text { The Author(s). Published by S. Karger AG, Basel } \\
\text { www.karger.com/cpb }\end{array}$ \\
\hline
\end{tabular} \\ Chen et al.: Sulforaphane Inhibits Pancreatic Cancer}

\section{Materials and Methods}

\section{Cell culture and reagents}

Panc-1 and MiaPaca-2, two different pancreatic cancer cell lines, were obtained from the Cell Bank of the Chinese Academy of Sciences (Shanghai, China). Human Umbilical Vein Endothelial Cells (HUVECs) were kindly provided by Dr. Chang Liu (Medical College, Xi'an Jiaotong University). Cells were cultured in Dulbecco's modified Eagle's medium (DMEM) (Gibco BRL Co. Ltd., USA) supplemented with 10\% heatinactivated fetal bovine serum (FBS), $100 \mu \mathrm{g} / \mathrm{ml}$ streptomycin and $100 \mathrm{U} / \mathrm{ml}$ ampicillin at $37^{\circ} \mathrm{C}$ under $5 \%$ $\mathrm{CO}_{2}$ and $95 \%$ air. SFN and compound C were purchased from Sigma (St. Louis, MO, USA). Antibodies used in this study were purchased from Cell Signaling Technology. 2'-7'-Dichlorofluorescein diacetate (DCF-DA) was purchased from Beyotime Biotechnology. Dimethyl sulfoxide (DMSO) and 3-(4, 5-dimethylthiazol-2-yl)-2, 5-diphenyl tetrazolium bromide (MTT) were obtained from Sigma.

\section{MTT assay}

A total of $5 \times 10^{3}$ cells per well were seeded in a 96-well plate and cultured in $10 \%$ FBS medium. The next day, the cells were starved with $1 \%$ FBS medium for approximately 12 hours and then treated in $10 \%$ FBS medium containing different concentrations of SFN or glucose. At the indicated time points $(24,48$, and 72 hours), $20 \mu \mathrm{l}$ of MTT solution ( $5 \mathrm{mg} / \mathrm{ml}$ dissolved in phosphate-buffered saline, PBS) was added to each well. After the cells were incubated for approximately 4 hours at $37^{\circ} \mathrm{C}$, they were retreated with $200 \mu \mathrm{l}$ of DMSO. Ten minutes later, the optical density (OD) value at $490 \mathrm{~nm}$ was detected using a microplate reader (BIO-TEC Inc, VA).

\section{Apoptosis assay}

Cell apoptosis was detected by flow cytometry with an Annexin V-FITC/7-AAD apoptosis detection kit (Becton, Dickinson and Company, NJ, USA), according to the manufacturer's instructions. In brief, $1 \times 10^{5}$ cells were seeded in a 6-well plate, starved overnight and treated with different concentrations of glucose or SFN in serum-starved medium. After 48 hours, the cells were washed twice with PBS, trypsinized, and then resuspended in $1 \times$ binding buffer. Ten minutes after incubation with PE Annexin V and 7-AAD, the percentage of apoptotic cells was detected with a flow cytometer (BD Biosciences, USA) according to the manufacturer's instructions.

\section{Colony formation assay}

Briefly, $1 \times 10^{3}$ cells were evenly seeded in a $60-\mathrm{mm}$ dish and cultured overnight to allow cells to adhere. Then, the cells were treated with a different intervention culture medium containing $10 \%$ FBS. Two days later, the medium was replaced with drug-free medium. Ten days after the cells were seeded, colonies formed. After the cells were washed twice with PBS, they were fixed with $4 \%$ paraformaldehyde and further stained with $0.1 \%$ crystal violet staining solution. Images were collected with a Nikon camera, and the colony number was counted.

\section{Cell migration and invasion assays}

Cell migration was assessed by a wound-healing assay. Cancer cells were pretreated with SFN under low or high glucose conditions and then scratched using a $200 \mu$ pipette tip. Then, the cells were washed with PBS 3 times and cultured in serum-starved medium. Images were taken with a microscope (Nikon Instruments Inc.). A Matrigel Transwell assay was used to test cell invasion ability as described in the previous study [22]. Chambers were pre-coated with Matrigel (BD Biosciences, USA). Approximately $5 \times 10^{4}$ cells were pretreated with SFN under low or high glucose conditions, resuspended in $200 \mu \mathrm{l}$ of serumstarved medium and seeded into the upper chamber. Then, $500 \mu \mathrm{l}$ of DMEM culture medium containing $10 \%$ FBS was added to the lower chamber. After $24 \mathrm{~h}$, the cells were washed with PBS 3 times, fixed with $4 \%$ paraformaldehyde, and stained with crystal violet. Images were taken at a magnification of 200x.

\section{Western blotting}

Cells were harvested and lysed in RIPA lysis buffer containing protease and phosphatase inhibitors. Total protein was electrophoresed in a 10\% SDS-PAGE gel and then transferred to PVDF membranes, which were then blocked with $10 \%$ milk in PBST. The membranes were incubated with specific primary antibodies 


\section{Cellular Physiology Cell Physiol Biochem 2018;50:1201-1215 \begin{tabular}{ll|l} 
and Biochemistry & $\begin{array}{l}\text { DOI: 10.1159/000494547 } \\
\text { Published online: 24 October } 2018\end{array}$ & $\begin{array}{l}\text { (c) } 2018 \text { The Author(s). Published by S. Karger AG, Basel } \\
\text { www.karger.com/cpb }\end{array}$ \\
\hline
\end{tabular} \\ Chen et al.: Sulforaphane Inhibits Pancreatic Cancer}

overnight at $4^{\circ} \mathrm{C}$. After the membranes were washed with PBST, they were incubated with HRP-conjugated secondary antibodies for 2 hours at room temperature and then washed three times again. Immunoreactive bands were visualized using an enhanced chemiluminescence kit. Images were captured by a ChemiDoc XRS imaging system (Bio-Rad, USA).

Detection of intracellular Reactive oxygen species (ROS)

Intracellular ROS was measured using a Reactive Oxygen Species Assay Kit. After the cells were washed with PBS, they were pretreated with SFN under low or high glucose conditions and then incubated with 10 $\mu \mathrm{M}$ DCF-DA in serum-starved DMEM for approximately 30 minutes. Cells were then washed 3 times with PBS and lysed with $1 \mathrm{ml}$ of PIPA buffer. ROS production was then analyzed by fluorometric analysis at 510 $\mathrm{nm}$. The final results were normalized to the total protein content.

\section{Transgenic pancreatic cancer mouse and nude mice}

Transgenic pancreatic cancer mouse was introduced as previous described [23]. $20 \mathrm{Kras}^{\mathrm{G} 12 \mathrm{D}}$; Pdx1-Cre (KC) mouse and $16 \mathrm{Kras}^{\mathrm{G} 12 \mathrm{D}}$; Trp53 $3^{\mathrm{lox} / \mathrm{lox}} \mathrm{Pdx} 1$-Cre (KPC) mouse were randomly divided into 2 groups; Control group and SFN treatment group. SFN intervention group were administered with SFN at a dose of $50 \mathrm{mg} / \mathrm{kg}$ through intraperitoneal injection every other day. KPC mouse were monitored for up to 120 days and then were killed for sample collection. KC mouse were killed every month to show the pathological change. Fiveweek-old nude mice were randomly divided into three groups: control group, hyperglycemia group, and hyperglycemia plus SFN intervention group. Mice in the last two groups were injected with streptozotocin $(150 \mathrm{mg} / \mathrm{kg})$ intravenously to induce hyperglycemia. One week later, $1 \times 10^{6}$ Panc- 1 pancreatic cancer cells were subcutaneously implanted on the right limb of mice. After one week, mice in the hyperglycemia and SFN intervention group were administered SFN at a dose of $50 \mathrm{mg} / \mathrm{kg}$ through intraperitoneal injection every other day. Tumor size was monitored every three days, and tumor size was calculated using the formula width $^{2} \times$ length $/ 2$. At the end of our experiment, mice were weighed and euthanized, and tumor weights were measured. In addition, the tumors were then fixed in $4 \%$ paraformaldehyde, and immunohistochemical staining was performed.

\section{Statistical analysis}

All results are presented as the mean \pm SD of multiple independent experiments. Student's $t$ test was used to analyze the difference between two groups. A Kruskal-Wallis one-way ANOVA followed by Dunn's multiple comparison tests was used to analyze the difference among more than two groups. All statistical analyses were performed with GraphPad Prism 7. $P<0.05$ was defined as statistically significant.

\section{Results}

SFN inhibits the proliferation of pancreatic cancer cells under both normal and high glucose conditions

We examined the effect of SFN on the proliferation of pancreatic cancer cells. Two different pancreatic cancer cell lines were treated with increasing concentrations of SFN, from $1 \mu \mathrm{M}$ to $100 \mu \mathrm{M}$. HUVECs were selected as the normal control. Cell viability was assessed by MTT assay at different time points $(24 \mathrm{~h}, 48 \mathrm{~h}$, and $72 \mathrm{~h})$. SFN inhibited the growth of pancreatic cancer cells in a time- and dose-dependent manner (Fig. 1A and 1B). However, we did not observe any significant inhibitory effects on HUVECs at a concentration of less than $20 \mu \mathrm{M}$ (Fig. 1C). In this study, we selected $20 \mu \mathrm{M}$ SFN for subsequent experiments.

Previous study found that hyperglycemia can accelerate the growth of pancreatic cancer. We further determined whether SFN can influence high glucose-induced promotion of the growth of pancreatic cancer cells. In our experiment, $25 \mathrm{mM}$ glucose can greatly promote cell growth compared with $5.5 \mathrm{mM}$ glucose (Fig. 1D and 1E). However, this effect was reversed by SFN intervention (Fig. 1D and 1E). We also used EdU staining to show these effects more intuitively (Fig. 1F and $1 \mathrm{G}$ ). These results suggest that SFN can inhibit the proliferation of pancreatic cancer cells cultured in both low and high glucose media. 


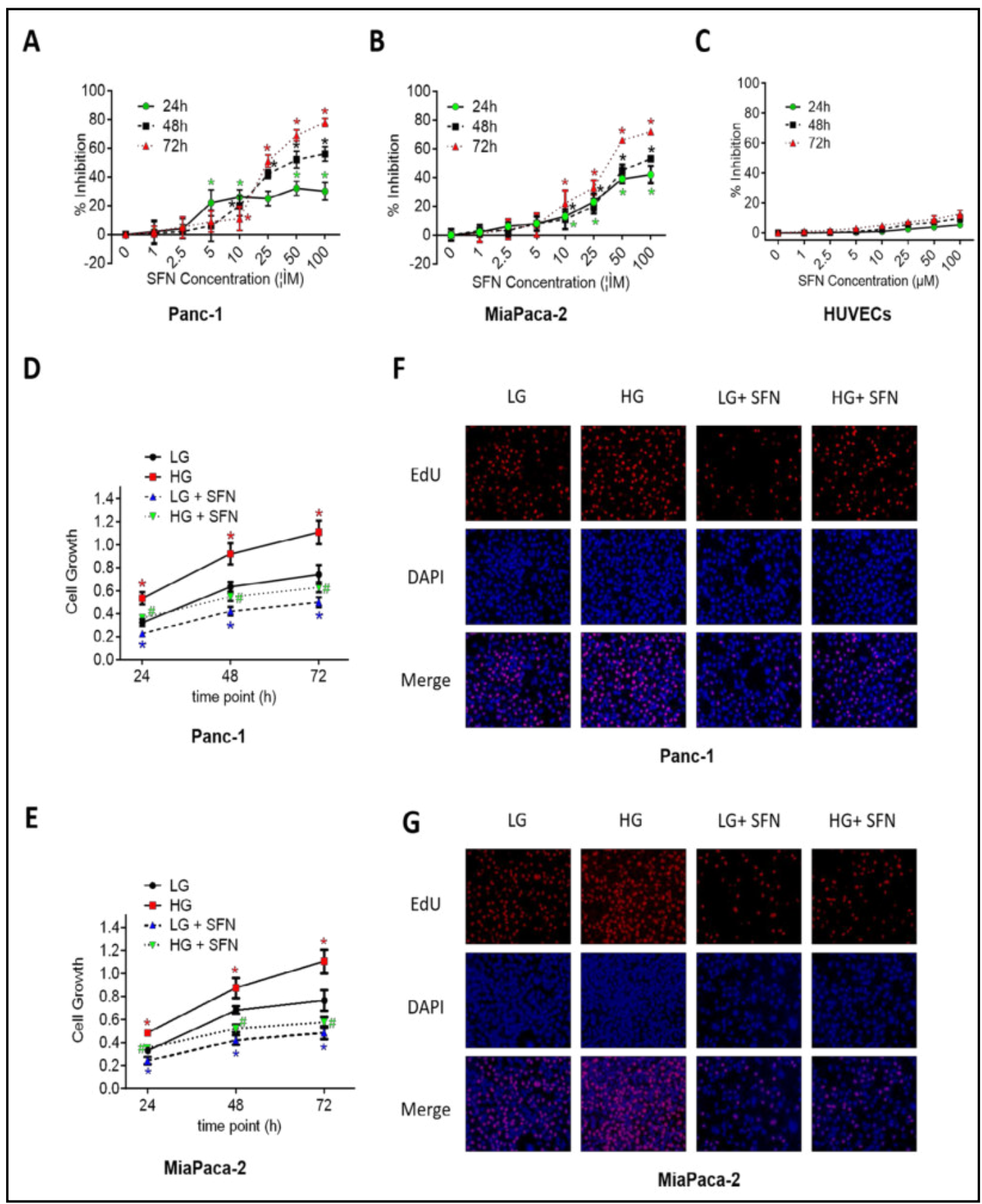

Fig. 1. Sulforaphane inhibits the proliferation of pancreatic cancer cells both under low and high glucose conditions. (A-C) Pancreatic cancer cells (Panc-1 and MiaPaca-2 cells) and human normal cells (HUVECs) were treated with increasing concentration of SFN, cell viability was tested by MTT assay at indicated time points $(24,48$, and $72 \mathrm{~h}) .{ }^{*} \mathrm{P}<0.05$ when compared with untreated control group. (D, E) Panc- 1 and MiaPaca-2 cells were left with or without SFN under low glucose (LG) or high glucose (HG) medium, and cell viability was assessed by MTT at the indicated time points $(24,48$, and $72 \mathrm{~h})$. ${ }^{*} \mathrm{P}<0.05$ when compared with low glucose group, $\# \mathrm{P}<0.05$ when compared with high glucose group. (F, G) Panc- 1 and MiaPaca-2 cells were left with or without SFN under low or high glucose medium, and cell viability was assessed by EdU staining at $48 \mathrm{~h}$. 
SFN inhibits clone formation, apoptosis and migration of pancreatic cancer

We next determined the role of SFN on clone formation. A decreased number of colonies was found in SFN group, compared with the control group. When cells were cultured under high glucose conditions, the number of colonies increased significantly. However, this promotion effect was reversed by SFN treatment (Fig. 2A and 2B). We further estimated apoptotic cell death using flow cytometric analyses. As shown in Fig. 2C and 2D, a smaller

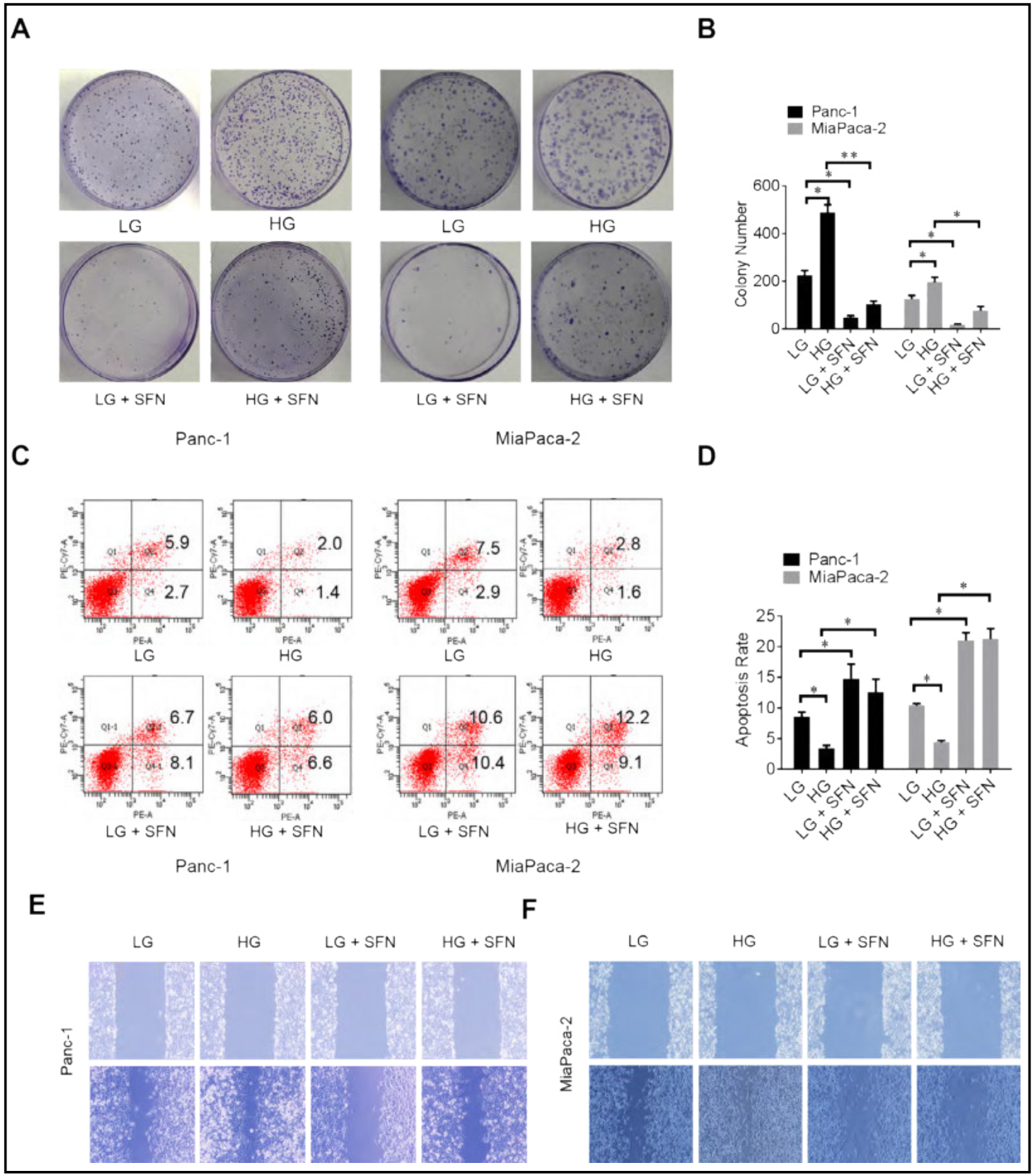

Fig. 2. Sulforaphane intervention decreases colony formation, cell migration and promotes the apoptosis. (A) Pancreatic cancer cells (Panc-1 and MiaPaca-2 cells) were exposed to low or high glucose conditions with or without SFN; a representative image of colony formation is shown from three independent experiments. (B) Colony numbers of various groups shown in graph (A) were quantified. (C) Pancreatic cancer cells were exposed to various conditions for $24 \mathrm{~h}$, and cell apoptosis was detected by flow cytometry. (D) Cell apoptosis of various groups shown in graph (C) were quantified. (E, F) Panc-1 and MiaPaca-2 cells were starved for 24 $\mathrm{h}$ and then treated under different conditions, cell migratory ability was assessed by wound healing assay $(100 \times) .{ }^{*} \mathrm{P}<0.05,{ }^{* *} \mathrm{P}<0.01$.

\section{KARGER}




\section{Cellular Physiology Cell Physiol Biochem 2018;50:1201-1215

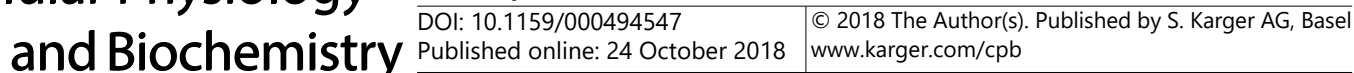 \\ Chen et al.: Sulforaphane Inhibits Pancreatic Cancer}

percentage of apoptotic cells was found in high glucose medium compared with that in low glucose medium. In addition, SFN could still greatly promote cell apoptosis, even in high glucose medium (Fig. 2C and 2D). Further, we found SFN inhibits the migratory ability of pancreatic cancer cells in both low and high glucose condition (Fig. 2E and 2F). These data suggest that SFN may be a very potent drug for pancreatic cancer.

\section{SFN decreases cell invasion ability through ROS induced EMT}

Cell invasion is the first step for cancer migration. Here, we explored the effect of SFN on the invasion ability of pancreatic cancer cells. The number of invading cells in the lower chamber decreased greatly when SFN was added, regardless of the glucose concentration in the medium (Fig. 3A and 3B). EMT is related to cell invasion. We found that high glucose increased protein levels of mesenchymal markers, such as $\mathrm{N}$-cadherin and vimentin. Meanwhile, decreased epithelial marker E-cadherin at protein level compared with normal glucose. In contrast, SFN clearly inhibited the mesenchymal markers and upregulated the epithelial markers (Fig. 3C and 3D). Intracellular ROS plays an important role in cancer progression, especially in cancer invasion. Here, we found that high glucose conditions increased the intracellular level of ROS, while SFN dramatically boosted these effects (Fig. $3 \mathrm{E}$ and $3 \mathrm{~F}$ ). We wonder whether suppress ROS production could rescue cell invasion. Using N-acetylcysteine (NAC), cellular ROS production was reduced dramatically (Fig. 3G). Meanwhile, the invaded cells number in NAC and SFN co-treatment group increased a lot compared with SFN group (Fig. 3H and 3I). These findings indicate that SFN inhibits cell invasion likely through ROS-EMT pathway.

AMPK and Nrf2/HO-1 activation mediate SFN-induced suppression of pancreatic cancer cells in normal and high glucose media

SFN has been found to be a potent Nrf2 activator, so we detected the changes in Nrf2 expression in our experiment. Compared with the low glucose group, high glucose decreased the nuclear localization of Nrf2 and increased the cytoplasmic expression of Nrf2. In contrast, SFN treatment promoted the nuclear localization of Nrf2 in both normal and high glucose media, as confirmed by western blotting and immunofluorescence. The expression of the downstream target HO-1 was also strengthened by SFN intervention (Fig. 4A-4D). Hyperglycemia-induced AMPK repression plays an important role in the promotion of pancreatic cancer. Here, we also verified that SFN can substantially activate the AMPK signaling pathway (Fig. 4E and 4F). These findings suggest that AMPK signaling and Nrf2/ HO-1 signaling are involved in the protective effect of SFN in pancreatic cancer.

Inhibition of AMPK suppresses Nrf2 expression and rescues pancreatic cancer cell invasion ability

To further verify the role of AMPK signaling, we used compound C, a small molecular inhibitor of the AMPK signaling pathway, in subsequent experiments. As can be seen, $20 \mu \mathrm{M}$ compound $\mathrm{C}$ obviously inhibited p-AMPK expression. The nuclear localization of Nrf2 was also weakened by compound $\mathrm{C}$ intervention, accompanied by increased cytoplasmic Nrf2 expression (Fig. 5A and 5B). Pretreatment with compound C suppressed SFN-induced AMPK activation and $\mathrm{Nrf} 2 / \mathrm{HO}-1$ expression under high glucose conditions in both MiaPaca-2 and Panc-1 cells, suggesting that Nrf2/HO-1 may be a downstream target of AMPK signaling (Fig. 5C). Next, we determined whether AMPK inhibition can rescue the invasion ability of the cells. As shown in Fig. 5D and 5E, the number of invading cells was reduced by SFN treatment. However, when cells were pretreated with compound $\mathrm{C}$, the number of invaded cells significantly increased, although slightly less than the control group. 


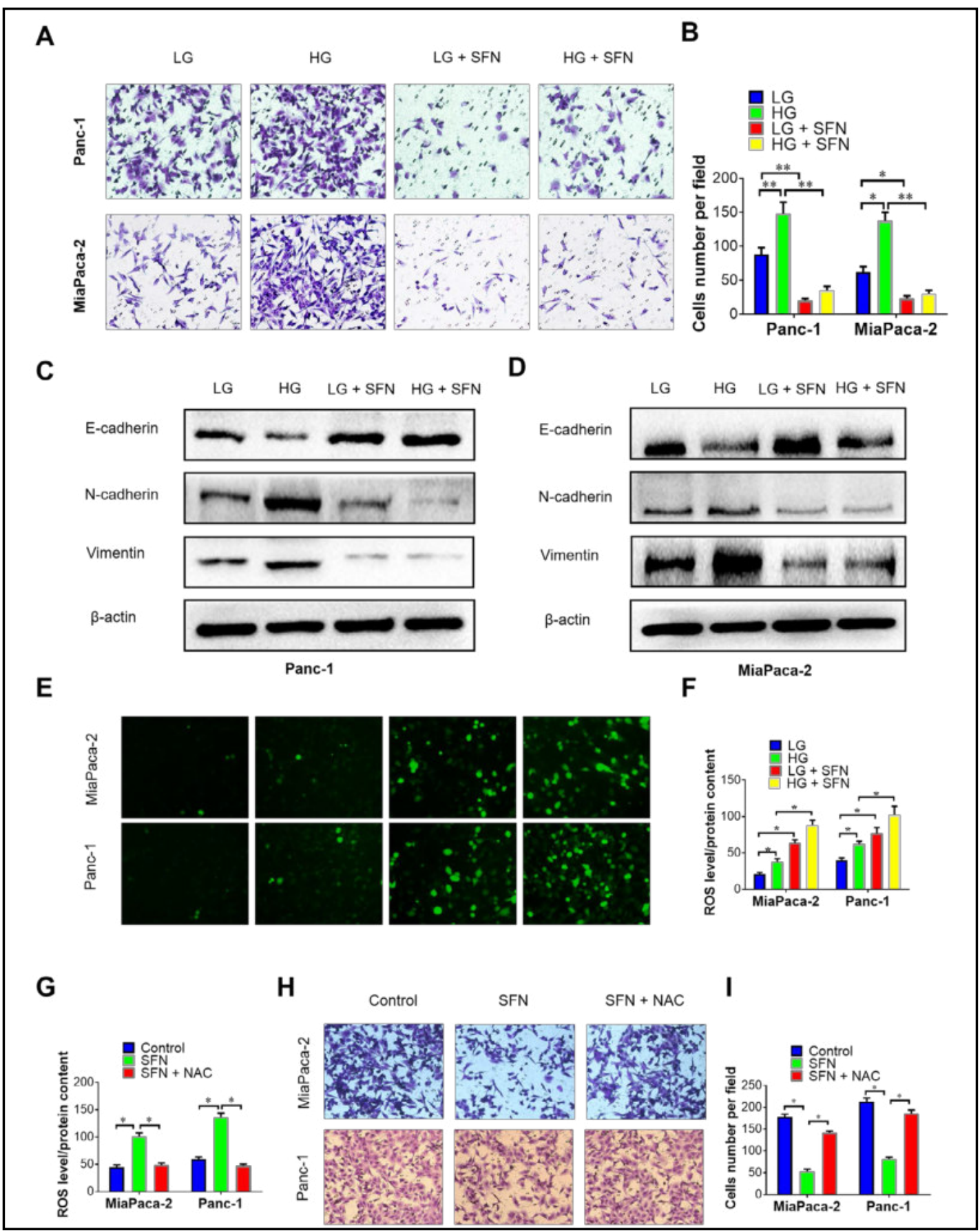

Fig. 3. Sulforaphane inhibits the invasion ability of cancer cells through ROS-EMT pathway. (A) $5 \times 10^{4}$ cells Panc-1 or MiaPaca-2 in $200 \mu$ l serum-starved DMEM medium containing low or high glucose with or without SFN were seeded on the upper chamber, and cell invasion was assessed by Transwell assay. Representative images are shown (200x). (B) The relative cell invasion ability shown in graph (A) was quantified. (C, D) EMT-related protein was analyzed by Western blotting. (E) ROS production was detected by fluorescence microscopy, and representative images are shown (200x). (F, G) Cells treated in different groups were incubated with DCFDA for 30 minutes, washed with PBS three times and lysed with RIPA lysis buffer. ROS production was tested by fluorimetry at $510 \mathrm{~nm}$, and the result was normalized to total protein levels. $(\mathrm{H})$ $5 \times 10^{4}$ cells Panc- 1 or MiaPaca- 2 in $200 \mu$ l serum-starved DMEM medium containing high glucose were seeded on the upper chamber, and cell invasion was assessed by Transwell assay (200x). (I)The relative cell invasion ability shown in graph $(\mathrm{H})$ was quantified. $* \mathrm{P}<0.05$.

\section{KARGER}


A

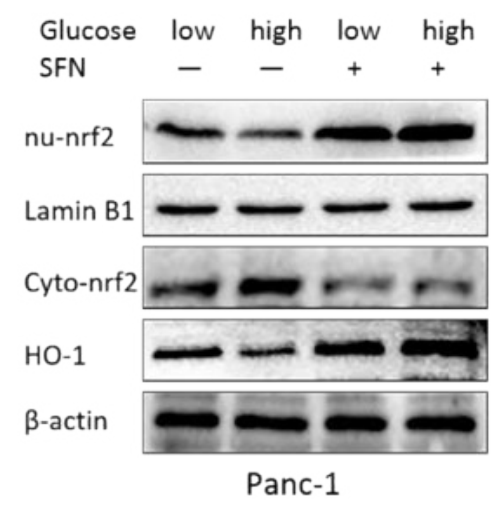

C

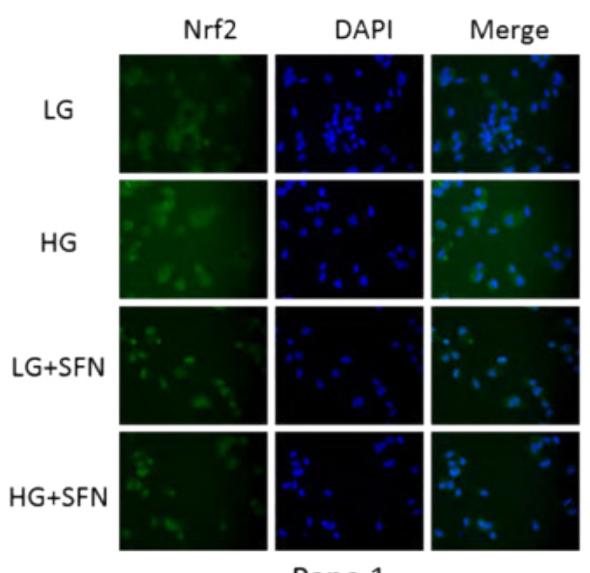

E

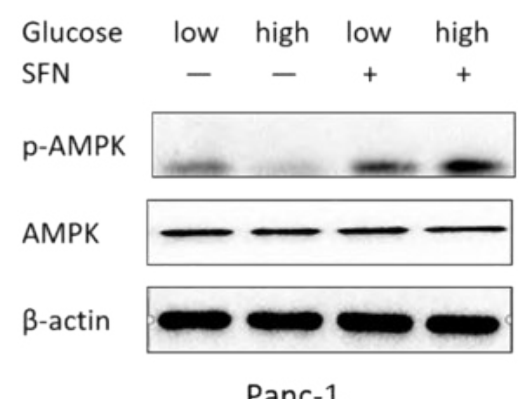

B

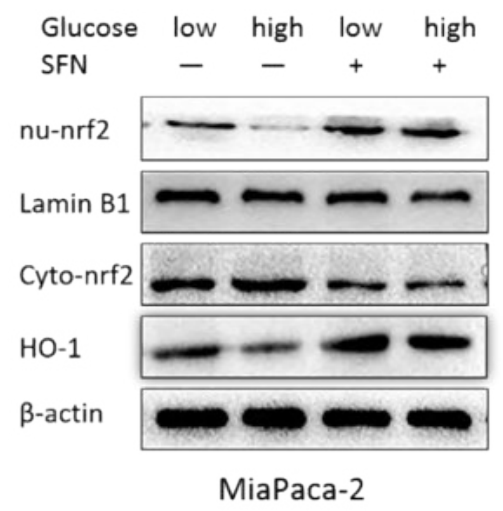

D

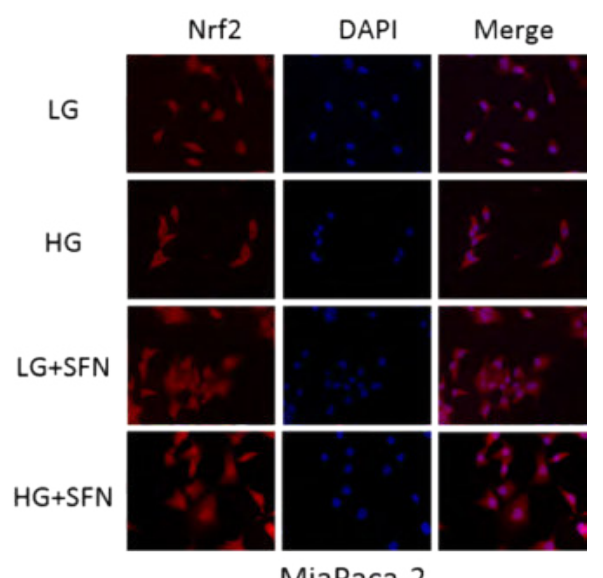

$\mathbf{F}$

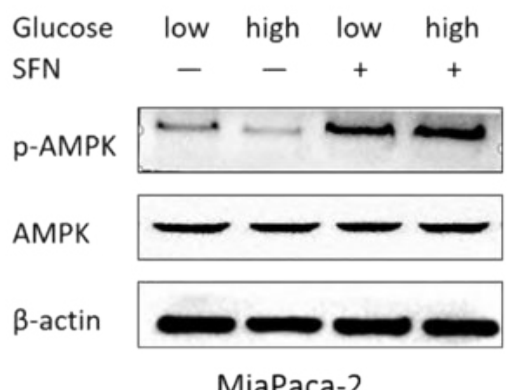

MiaPaca-2

Fig. 4. AMPK signaling and $\mathrm{Nrf2/HO}-1$ activation mediate $\mathrm{SFN}$-induced suppression of pancreatic cancer cells. (A-B) Effect of SFN on the protein levels of Nrf2 and HO-1 after $48 \mathrm{~h}$ intervention as detected by Western blotting. (C-D) Representative images of immunofluorescence show the translocation of Nrf2 in various groups (400×). (E-F) Effect of SFN on the protein level of p-AMPK after $48 \mathrm{~h}$ intervention as detected by Western blotting.

SFN inhibits tumor growth in vivo

Next, we determined the role of SFN in vivo. BALb/c mice were subcutaneously injected with $1 \times 10^{6}$ Panc- 1 pancreatic cancer cells on the right limb. Mice were randomly divided into three groups, and the detailed treatment can be seen in the Materials and Methods. At the end of our experiment, mice with hyperglycemia displayed larger tumor volume and tumor weight at the end of our experiment. However, SFN treatment greatly inhibited 
A

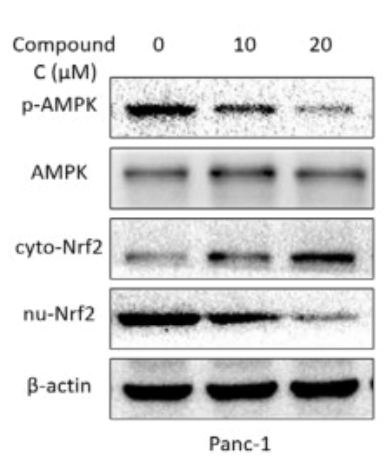

B
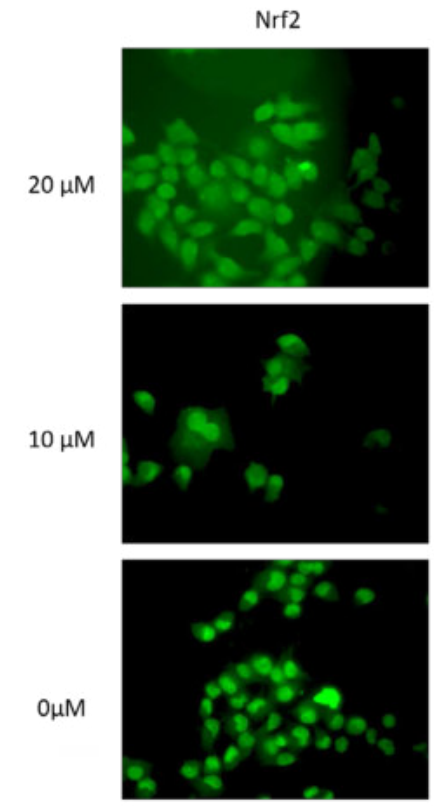

D

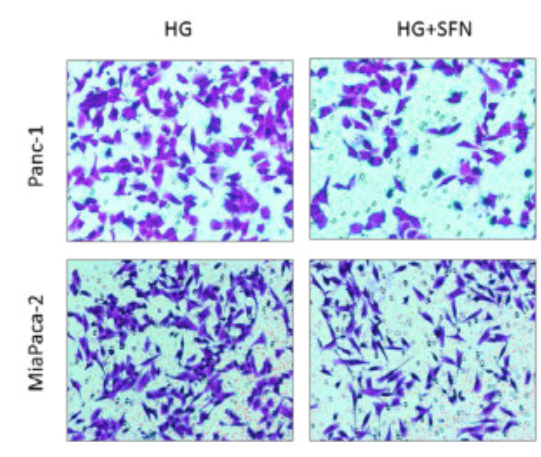

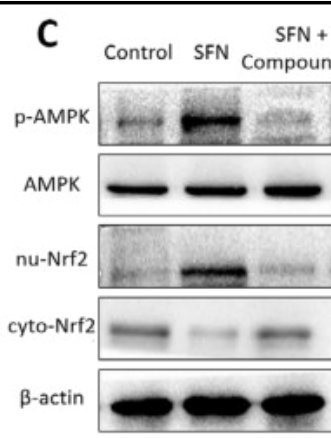

Panc-1

DAPI
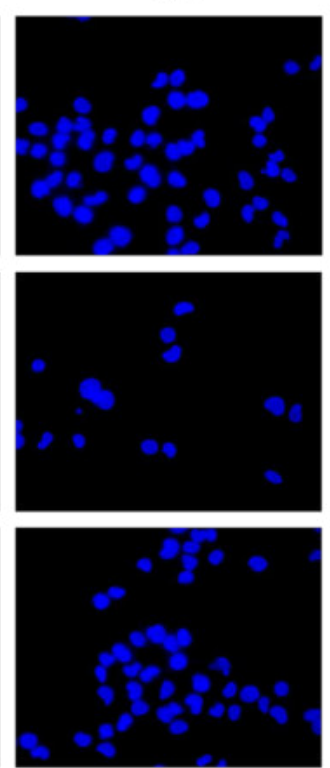

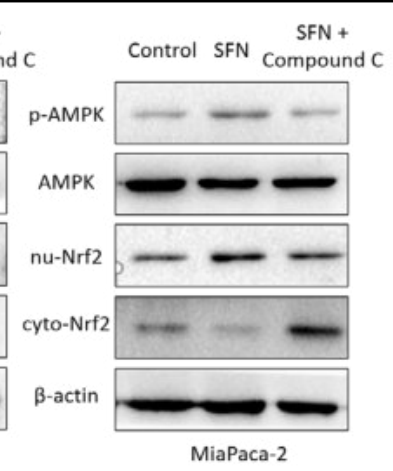

Merge
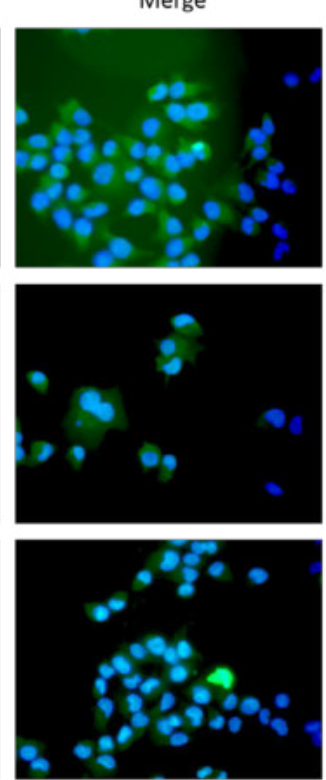

$\mathbf{E}$ $\mathrm{HG}+\mathrm{SFN}+$ Compound C
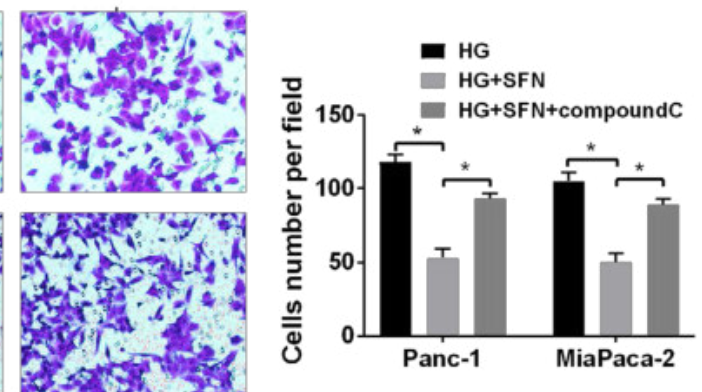

Fig. 5. Inhibition of AMPK suppresses nuclear Nrf2 expression and rescues pancreatic cancer cell invasion ability. (A) Pancreatic cancer cells were treated with different concentrations of compound C, and the protein levels of p-AMPK and nuclear or cytoplasm Nrf2 were detected by Western blotting. (B) Translocation of Nrf2, which was induced by compound C, was elucidated by immunofluorescence (400x). (C) Pancreatic cancer cells cultured in high glucose medium were treated with SFN with or without compound C, and the protein levels of p-AMPK and nuclear or cytoplasm Nrf2 were detected by Western blotting. (D) Inhibition of AMPK rescues the invasion ability of pancreatic cancer cells. Cells cultured under high glucose conditions were treated with SFN with or without compound C, and their invasion ability was assessed by Transwell assay $(200 \times)$. (E) The relative cell invasion ability of cells shown in graph (D) was quantified. ${ }^{*} \mathrm{P}<0.05$. 


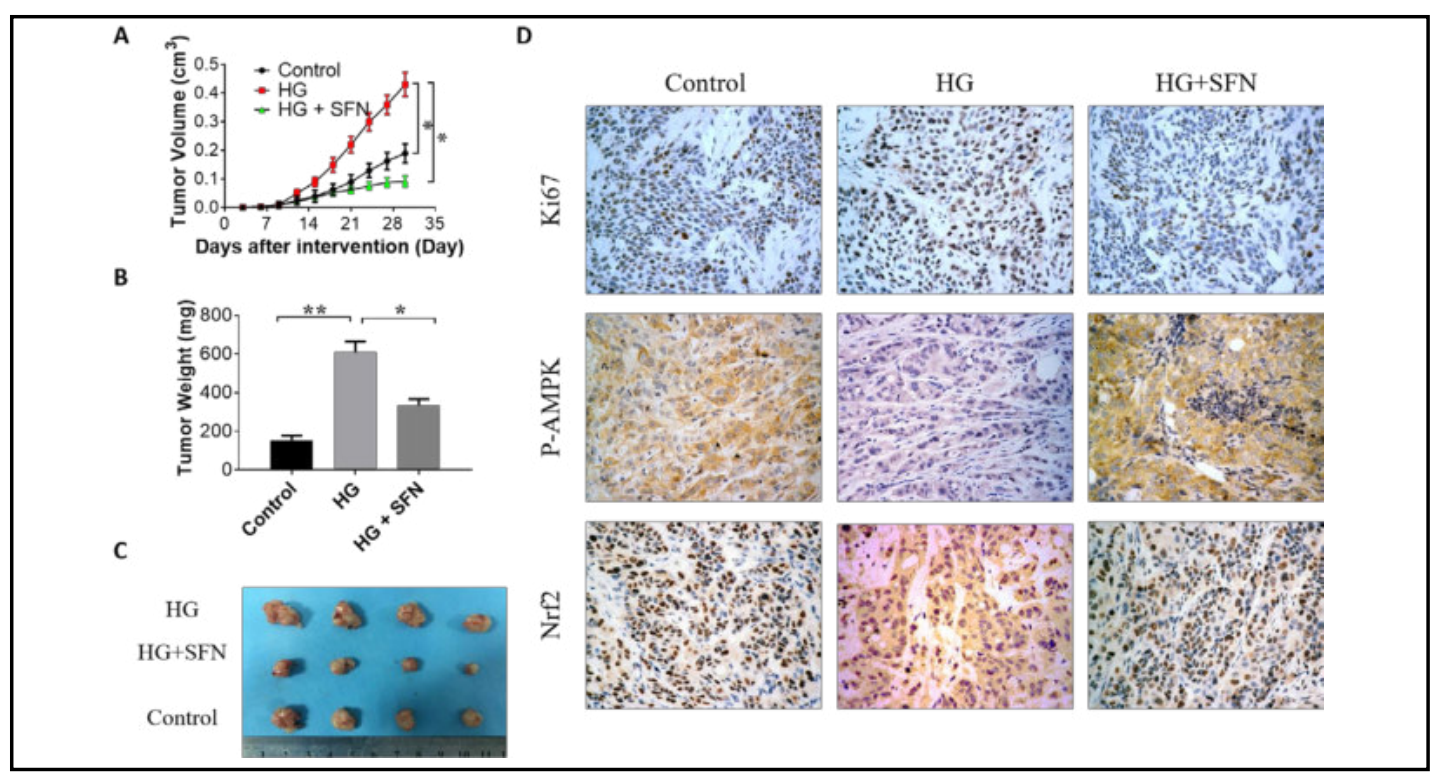

Fig. 6. Sulforaphane decreases tumor volume and tumor weight in BALb/c mice. (A) Tumor volume was calculated every 3 days after tumor implantation. Hyperglycemia can promote tumor growth, which could be inhibited by SFN. (B, C) SFN inhibits the increase in tumor weight at the end of our experiment. (D) Representative image of immunohistochemical staining for Ki-67, Nrf2 and p-AMPK in our three groups of mice $(400 \times) .{ }^{*} \mathrm{P}<0.05,{ }^{* *} \mathrm{P}<0.01$.

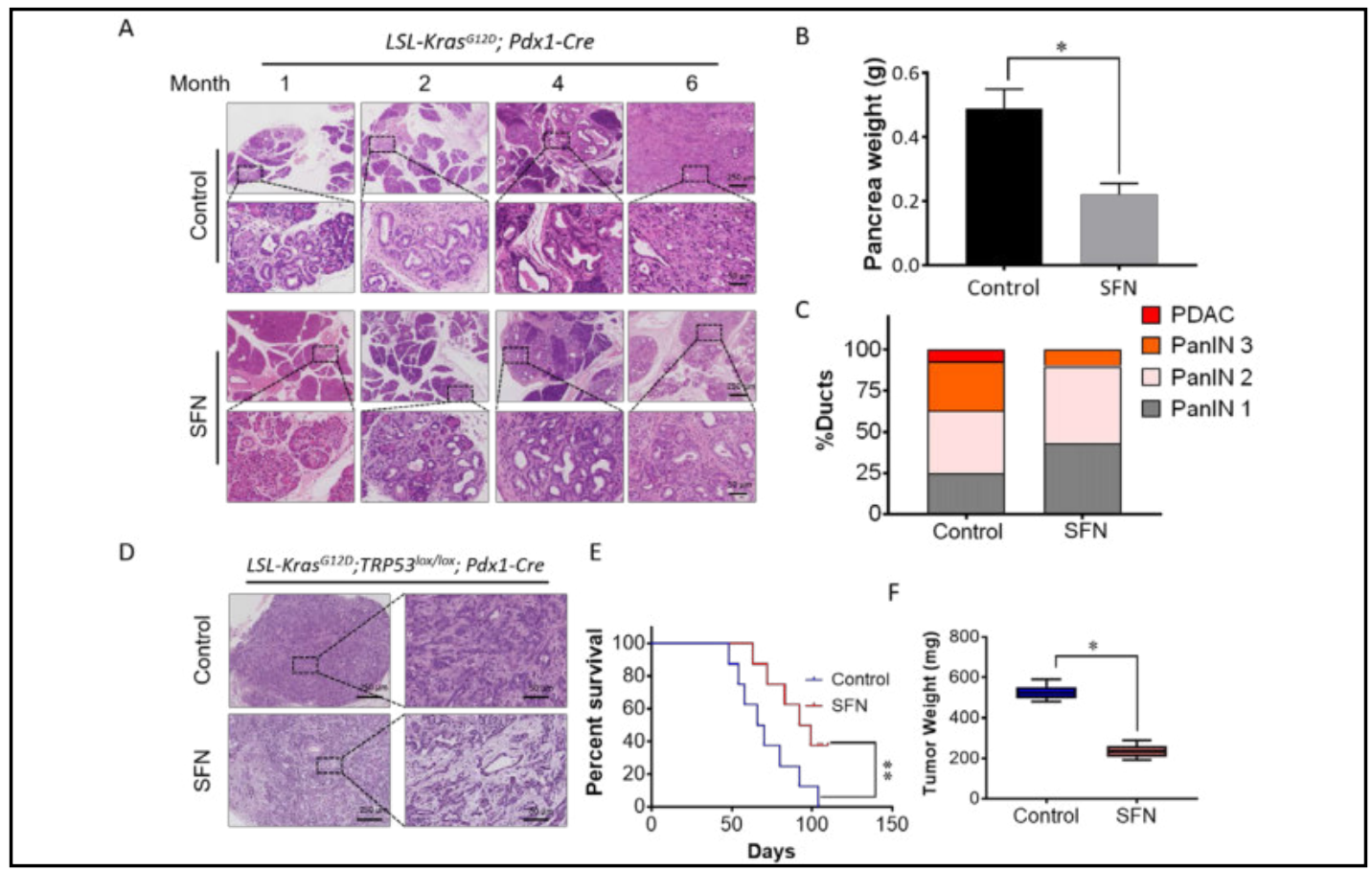

Fig. 7. SFN suppresses tumor progression in transgenic pancreatic cancer mouse model. (A) KC mouse were divided into control and SFN group. SFN intervention group were administered SFN at a dose of $50 \mathrm{mg} / \mathrm{kg}$ through intraperitoneal injection every other day. Mouse was killed every month to show the cancer lesions. Representative images at different time point after SFN intervention are shown. (B) Pancreas weight of mouse 6 months after intervention. (C) Quantification of the percentage of PanIN in 6 monoths old mouse, 5 months after SFN treatment. (D) Representative HE staining images of KPC mouse pancreas at the end of experiment. (E) Kaplan-Meier survival analysis of KPC mice treated with vehicle or SFN. (F) Tumor weight of KPC mouse in control and SFN group. ${ }^{*} \mathrm{P}<0.05,{ }^{* *} \mathrm{P}<0.01$. 


\section{Cellular Physiology Cell Physiol Biochem 2018;50:1201-1215 \begin{tabular}{l|l} 
DOI: 10.1159/000494547 & $\begin{array}{l}\text { O } 2018 \text { The Author(s). Published by S. Karger AG, Basel } \\
\text { www.karger.com/cpb }\end{array}$
\end{tabular} \\ Chen et al.: Sulforaphane Inhibits Pancreatic Cancer}

tumor growth, exhibiting a smaller tumor volume (Fig. 6A-6C). Moreover, we did not find any significant difference in the weight of mice in these three groups (data not shown). Immunohistochemistry also revealed a lower or weaker expression of Ki-67 when SFN was administered. Consistent with our in vitro study, immunohistochemistry also showed weaker expression of p-AMPK in mice with diabetes mellitus, which was reversed by SFN. Nrf2 nuclear localization was also strengthened by SFN treatment in vivo (Fig. 6D). These results imply that SFN may be a good choice for cancer therapy in vivo.

\section{SFN suppresses tumor progression in transgenic pancreatic cancer mouse model}

Genetically engineered pancreatic cancer mouse model can recapitulate the histopathological characteristics of PDAC in human patients. So it is a good choice to testify the therapeutic effect of cancer drugs. Here, we showed that SFN suppress PanIN lesions progression in KC mice at different time point (Fig. 7A). Five months after SFN treatment, the pancreas weight in SFN group is much lower than control group (Fig. 7B). We didn't find PDAC lesions in SFN group mouse (Fig. 7C). However, 7\% of the lesions in control group progressed into PDAC (Fig. 7C). Meanwhile, we also find a less percent of PanIN 3 lesions in SFN group (Fig. 7C). In KPC mouse, more ductal structure can be found in SFN group (Fig. 7D). Mouse accepted SFN intervention have a longer survival in our experiment (Fig. 7E). Moreover, the tumor weight is much lower in SFN group. These data suggest that SFN can suppress tumor progression and prolong cancer survival in vivo.

\section{Discussion}

Hyperglycemia has been regarded as both a risk factor and a consequence of pancreatic cancer, while the mechanism and therapy remain a challenge to resolve. In the current study, we showed for the first time that SFN can inhibit high glucose-induced pancreatic cancer progression. SFN treatment can not only inhibit cell proliferation and invasion but also induce cell apoptosis under high glucose conditions. Activation of AMPK signaling may be involved in SFN intervention, which further results in the translocation of Nrf2, leading to the reversion of EMT and suppression of pancreatic cancer growth. These data suggest that SFN may be an effective drug especially suited for patients with pancreatic cancer who are suffering from diabetes mellitus.

High glucose has a pernicious effect on normal human cells, inhibiting growth and inducing apoptosis [24]. However, in pancreatic cancer, we found that high glucose can exacerbate cell proliferation and invasion, while decreasing cell apoptosis. Traditional antipancreatic cancer drugs, such as gemcitabine and 5-fluorouracil, have a potent anti-cancer effect. However, these cancer drugs can also kill normal human cells, and their low specificity limits clinical application. In our study, we confirmed that SFN can substantially repress pancreatic cancer cell proliferation in a time- and concentration-dependent manner under normal or high glucose conditions, without (or with little) toxicity to normal human cells (HUVECs) at concentrations of less than $30 \mu \mathrm{mol} / \mathrm{L}$, indicating that it is an ideal drug for cancer therapy.

Distant metastasis of pancreatic cancer, the main cause of cancer-related death and tumor recrudescence, which was traditionally thought to be a late event in cancer progression, has recently been confirmed to begin at a very early stage, even during tumor initiation [25]. Pancreatic cancer cells can secrete macrophage migration inhibitor abundant exosomes, which will be absorbed in the liver and create a fibrotic microenvironment to tempt distant metastasis [25]. Notable studies have discovered that a high glucose environment can promote liver, gastric, breast, and pancreatic cancer progression [26]. EMT endows cells with mesenchymal properties, which strengthen the invasive and migratory capacity of cells, and EMT is considered the first step in metastasis. In our study, we found enhanced invasive and migratory capabilities when cells were cultured in high glucose conditions, followed by EMT 


\section{Cellular Physiology Cell Physiol Biochem 2018;50:1201-1215 \begin{tabular}{ll|l} 
DOl: 10.1159/000494547 & O 2018 The Author(s). Published by S. Karger AG, Basel \\
www.karger.com/cpb
\end{tabular} \\ Chen et al.: Sulforaphane Inhibits Pancreatic Cancer}

progression. However, SFN intervention dramatically damaged cell invasive and migratory capabilities and inhibited EMT progression.

ROS production is confirmed to play a vital role in cell invasion $[27,28]$. Pancreatic cancer is characterized by an uncommonly strong desmoplastic reaction and poor vascular density, which means that a hypoxic microenvironment and ROS may play a large role in cancer progression. ROS are a double-edged sword; low to intermediate levels of ROS production may promote tumor initiation and progression, while excessive ROS can restrain cell viability, promote cell apoptosis, induce cell autophagy, and even trigger cell death. ROS induction currently has become a focus for anti-cancer drug design and application [29]. Here, increased ROS generation was detected when cells were cultured in a high glucose medium, which may account for the enhanced invasive ability. However, an extremely high level of ROS was detected in SFN-treated cells, likely resulting in cell death, apoptosis and decreased invasion.

The role of NRF2 in cancer development has been highly controversial [30]. It was reported that accumulation of Nrf2 in cancer cells creates an environment conducive for cell growth and protects against oxidative stress, chemotherapeutic agents, and radiotherapy (This phenomenon has been termed the "dark side of Nrf2") [31, 32]. However, the anticancer effect of NRF2 has also been observed. SFN, as an effective activator of NRF2, can inhibit carcinogenesis in many animal models. Reduced tumor incidence was found in wild type mice after SFN intervention, but not in NRF2 Knockout mice [33, 34]. In addition to direct anti-inflammatory effects mediated through NRF2 signaling, SFN may impair the redoxsensitive DNA binding and transactivation of the pro-inflammatory transcription factor NF$\kappa B$ [35]. In this study, the anti-cancer effect of SFN in pancreatic cancer was confirmed by in vitro and in vivo experiments with transgenic pancreatic cancer mouse model. In any case, these are only laboratory evidence imply that SFN may be an effective drug especially suited for patients with pancreatic cancer who are suffering from diabetes mellitus.

It has been reported that SFN can combat cancer by multiple mechanisms, including induce Nrf2 activation [36], epigenetic modulation of oncogenic MicroRNA-21 and human telomerase reverse transcriptase (hTERT) [37], inhibition of histone deacetylase (HDAC) [38] and NF- $\kappa B$ [39]. However, its anti-tumor effect on pancreatic cancer, especially under high glucose conditions, has not been investigated. Our results indicated that SFN inhibited high glucose-induced progression of pancreatic cancer via inducing Nrf2 and AMPK activation. Whether other mechanisms were involved in the anti-cancer effect of SFN in pancreatic cancer need further study.

\section{Conclusion}

In summary, our present study demonstrated that SFN can effectively prevent the high glucose-induced malignant behavior of pancreatic cancer in vivo and in vitro. Excessive ROS production that further induces apoptosis and inhibits migration and invasion may account for this preventive effect. Oxidative stress caused by SFN triggered the subcellular localization of Nrf2, which is mediated by AMPK signaling, and was verified to play an important role in this process. Collectively, SFN may be an effective choice for pancreatic cancer therapy, especially for patients with hyperglycemia.

\section{Abbreviations}

SFN (Sulforaphane); HUVECs (Human Umbilical Vein Endothelial Cells); LG (low glucose); HG (high glucose); EMT (epithelial-mesenchymal transition); Nrf2 (nuclear factor erythroid 2-related factor 2); DMSO (dimethyl sulfoxide); ROS (Reactive oxygen species); NAC (N-acetylcysteine); hTERT (human telomerase reverse transcriptase); HDAC (histone deacetylase). 


\section{Cellular Physiology Cell Physiol Biochem 2018;50:1201-1215 and Biochemistry Published \begin{tabular}{l|l} 
DOI: 10.1159/000494547 & $\begin{array}{l}\text { (c) } 2018 \text { The Author(s). Published by S. Karger AG, Basel } \\
\text { www.karger.com/cpb }\end{array}$
\end{tabular} \\ Chen et al.: Sulforaphane Inhibits Pancreatic Cancer}

\section{Acknowledgements}

This work was supported by grants from the National Natural Scientific Foundation of China (81572734, 81672434, 81702916) and grant from the Clinical Innovation Funds of the First Affiliated Hospital of Xi'an Jiaotong University (No. 13ZD17).

\section{Disclosure Statement}

The authors declare no conflict of interest.

\section{References}

1 Siegel RL, Miller KD, Jemal A: Cancer statistics, 2018. CA Cancer J Clin 2018;68:7-30.

-2 Lee JC, Ahn S, Cho IK, Lee J, Kim J, Hwang JH: Management of recurrent pancreatic cancer after surgical resection: a protocol for systematic review, evidence mapping and meta-analysis. BMJ Open 2018;8:e017249.

- 3 Roe JS, Hwang CI, TDD S, Milazzo JP, Lee EJ, Da SB, Maiorino L, Tiriac H, Young CM, Miyabayashi K, Filippini D, Creighton B, Burkhart RA, Buscaglia JM, Kim EJ, Grem JL, Lazenby AJ, Grunkemeyer JA, Hollingsworth MA, Grandgenett PM, Egeblad M, Park Y, Tuveson DA, Vakoc CR: Enhancer Reprogramming Promotes Pancreatic Cancer Metastasis. Cell 2017;170:875-888.e20.

4 Jemal A, Bray F, Center MM, Ferlay J, Ward E, Forman D: Global cancer statistics. CA Cancer J Clin 2011;61:69-90.

5 Li D, Tang H, Hassan MM, Holly EA, Bracci PM, Silverman DT: Diabetes and risk of pancreatic cancer: a pooled analysis of three large case-control studies. Cancer Causes Control 2011;22:189-197.

6 Lu Y, García RLA, Malgerud L, González-Pérez A, Martín-Pérez M, Lagergren J, Bexelius TS: New-onset type 2 diabetes, elevated HbA1c, anti-diabetic medications, and risk of pancreatic cancer. $\mathrm{Br} J$ Cancer 2015;113:1607-1614.

7 Pannala R, Leirness JB, Bamlet WR, Basu A, Petersen GM, Chari ST: Prevalence and clinical profile of pancreatic cancer-associated diabetes mellitus. Gastroenterology 2008;134:981-987.

-8 Bosetti C, Rosato V, Li D, Silverman D, Petersen GM, Bracci PM, Neale RE, Muscat J, Anderson K, Gallinger S, Olson SH, Miller AB, Bas BH, Scelo G, Janout V, Holcatova I, Lagiou P, Serraino D, Lucenteforte E, Fabianova E, et al.: Diabetes, antidiabetic medications, and pancreatic cancer risk: an analysis from the International Pancreatic Cancer Case-Control Consortium. Ann Oncol 2014;25:2065-2072.

$>9$ Li D, Yeung SC, Hassan MM, Konopleva M, Abbruzzese JL: Antidiabetic therapies affect risk of pancreatic cancer. Gastroenterology 2009;137:482-488.

10 Tang J, Li Z, Lu L, Cho CH: $\beta$-Adrenergic system, a backstage manipulator regulating tumour progression and drug target in cancer therapy. Semin Cancer Biol 2013;23:533-542.

-11 Yanaka A, Sato J, Ohmori S: Sulforaphane protects small intestinal mucosa from aspirin/NSAID-induced injury by enhancing host defense systems against oxidative stress and by inhibiting mucosal invasion of anaerobic enterobacteria. Curr Pharm Des 2013;19:157-162.

12 Matsui T, Nakamura N, Ojima A, Nishino Y, Yamagishi SI: Sulforaphane reduces advanced glycation end products (AGEs)-induced inflammation in endothelial cells and rat aorta. Nutr Metab Cardiovasc Dis 2016;26:797-807.

13 Atwell LL, Beaver LM, Shannon J, Williams DE, Dashwood RH, Ho E: Epigenetic Regulation by Sulforaphane: Opportunities for Breast and Prostate Cancer Chemoprevention. Curr Pharmacol Rep 2015;1:102-111.

14 Bauman JE, Zang Y, Sen M, Li C, Wang L, Egner PA, Fahey JW, Normolle DP, Grandis JR, Kensler TW, Johnson DE: Prevention of Carcinogen-Induced Oral Cancer by Sulforaphane. Cancer Prev Res (Phila) 2016;9:547557.

15 Liu KC, Shih TY, Kuo CL, Ma YS, Yang JL, Wu PP, Huang YP, Lai KC, Chung JG: Sulforaphane Induces Cell Death Through G2/M Phase Arrest and Triggers Apoptosis in HCT 116 Human Colon Cancer Cells. Am J Chin Med 2016;44:1289-1310.

16 Chen YJ, Myracle AD, Wallig MA, Jeffery EH: Dietary broccoli protects against fatty liver development but not against progression of liver cancer in mice pretreated with diethylnitrosamine. J Funct Foods 2016;24:57-62.

17 Shih YL, Wu LY, Lee CH, Chen YL, Hsueh SC, Lu HF, Liao NC, Chung JG: Sulforaphane promotes immune responses in a WEHI-3-induced leukemia mouse model through enhanced phagocytosis of macrophages and natural killer cell activities in vivo. Mol Med Rep 2016;13:4023-4029. 


\section{Cellular Physiology Cell Physiol Biochem 2018;50:1201-1215 \begin{tabular}{ll|l} 
and Biochemistry Published online: 24 October 2018 & $\begin{array}{l}\odot 2018 \text { The Author(s). Published by S. Karger AG, Basel } \\
\text { www.karger.com/cpb }\end{array}$ \\
\hline
\end{tabular} \\ Chen et al.: Sulforaphane Inhibits Pancreatic Cancer}

18 Rodova M, Fu J, Watkins DN, Srivastava RK, Shankar S: Sonic hedgehog signaling inhibition provides opportunities for targeted therapy by sulforaphane in regulating pancreatic cancer stem cell self-renewal. PLoS One 2012;7:e46083.

-19 Li SH, Fu J, Watkins DN, Srivastava RK, Shankar S: Sulforaphane regulates self-renewal of pancreatic cancer stem cells through the modulation of Sonic hedgehog-GLI pathway. Mol Cell Biochem 2013;373:217-227.

-20 Kallifatidis G, Rausch V, Baumann B, Apel A, Beckermann BM, Groth A, Mattern J, Li Z, Kolb A, Moldenhauer G, Altevogt P, Wirth T, Werner J, Schemmer P, Büchler MW, Salnikov AV, Herr I: Sulforaphane targets pancreatic tumour-initiating cells by NF-kappaB-induced antiapoptotic signalling. Gut 2009;58:949-963.

21 Rausch V, Liu L, Kallifatidis G, Baumann B, Mattern J, Gladkich J, Wirth T, Schemmer P, Büchler MW, Zöller M, Salnikov AV, Herr I: Synergistic activity of sorafenib and sulforaphane abolishes pancreatic cancer stem cell characteristics. Cancer Res 2010;70:5004-5013.

22 Duan W, Chen K, Jiang Z, Chen X, Sun L, Li J, Lei J, Xu Q, Ma J, Li X, Han L, Wang Z, Wu Z, Wang F, Wu E, Ma Q, Ma Z: Desmoplasia suppression by metformin-mediated AMPK activation inhibits pancreatic cancer progression. Cancer Lett 2017;385:225-233.

23 Chen K, Qian W, Jiang Z, Cheng L, Li J, Sun L, Zhou C, Gao L, Lei M, Yan B, Cao J, Duan W, Ma Q: Metformin suppresses cancer initiation and progression in genetic mouse models of pancreatic cancer. Mol Cancer 2017;16:131.

24 Jiao X, Li Y, Zhang T, Liu M, Chi Y: Role of Sirtuin3 in high glucose-induced apoptosis in renal tubular epithelial cells. Biochem Biophys Res Commun 2016;480:387-393.

-25 Costa-Silva B, Aiello NM, Ocean AJ, Singh S, Zhang H, Thakur BK, Becker A, Hoshino A, Mark MT, Molina H, Xiang J, Zhang T, Theilen TM, García-Santos G, Williams C, Ararso Y, Huang Y, Rodrigues G, Shen TL, et al.: Pancreatic cancer exosomes initiate pre-metastatic niche formation in the liver. Nat Cell Biol 2015;17:816826.

-26 Li W, Zhang L, Chen X, Jiang Z, Zong L, Ma Q: Hyperglycemia Promotes the Epithelial-Mesenchymal Transition of Pancreatic Cancer via Hydrogen Peroxide. Oxid Med Cell Longev 2016;2016:5190314.

-27 Yang Y, Deng S, Zeng Q Hu W, Chen T: Highly stable selenadiazole derivatives induce bladder cancer cell apoptosis and inhibit cell migration and invasion through the activation of ROS-mediated signaling pathways. Dalton Trans 2016;45:18465-18475.

-28 Zong L, Li J, Chen X, Chen K, Li W, Li X, Zhang L, Duan W, Lei J, Xu Q, Shan T, Ma Q, Sun H: Lipoxin A4 Attenuates Cell Invasion by Inhibiting ROS/ERK/MMP Pathway in Pancreatic Cancer. Oxid Med Cell Longev 2016;2016:6815727.

29 Prasad S, Gupta SC, Tyagi AK: Reactive oxygen species (ROS) and cancer: Role of antioxidative nutraceuticals. Cancer Lett 2017;387:95-105.

30 Sporn MB, Liby KT: NRF2 and cancer: the good, the bad and the importance of context. Nat Rev Cancer 2012;12:564-571.

-31 Moon EJ, Giaccia A: Dual roles of NRF2 in tumor prevention and progression: possible implications in cancer treatment. Free Radic Biol Med 2015;79:292-299.

32 Wang XJ, Sun Z, Villeneuve NF, Zhang S, Zhao F, Li Y, Chen W, Yi X, Zheng W, Wondrak GT, Wong PK, Zhang DD: Nrf2 enhances resistance of cancer cells to chemotherapeutic drugs, the dark side of Nrf2. Carcinogenesis 2008;29:1235-1243.

-33 Xu C, Huang MT, Shen G, Yuan X, Lin W, Khor TO, Conney AH, Kong AN: Inhibition of 7, 12-dimethylbenz(a) anthracene-induced skin tumorigenesis in C57BL/ 6 mice by sulforaphane is mediated by nuclear factor E2-related factor 2. Cancer Res 2006;66:8293-8296.

-34 Fahey JW, Haristoy X, Dolan PM, Kensler TW, Scholtus I, Stephenson KK, Talalay P, Lozniewski A: Sulforaphane inhibits extracellular, intracellular, and antibiotic-resistant strains of Helicobacter pylori and prevents benzo [a]pyrene-induced stomach tumors. Proc Natl Acad Sci U S A 2002;99:7610-7615.

-35 Heiss E, Herhaus C, Klimo K, Bartsch H, Gerhäuser C: Nuclear factor kappa B is a molecular target for sulforaphane-mediated anti-inflammatory mechanisms. J Biol Chem 2001;276:32008-32015.

-36 Dinkova-Kostova AT, Fahey JW, Kostov RV, Kensler TW: KEAP1 and Done? Targeting the NRF2 Pathway with Sulforaphane. Trends Food Sci Technol 2017;69:257-269.

-37 Martin SL, Kala R, Tollefsbol TO: Mechanisms for the Inhibition of Colon Cancer Cells by Sulforaphane through Epigenetic Modulation of MicroRNA-21 and Human Telomerase Reverse Transcriptase (hTERT) Down-regulation. Curr Cancer Drug Targets 2018;18:97-106.

-38 Juengel E, HHH E, Haferkamp A, Rutz J, Chun FK, Blaheta RA: Relevance of the natural HDAC inhibitor sulforaphane as a chemopreventive agent in urologic tumors. Cancer Lett 2018;435:121-126.

-39 Tortorella SM, Royce SG, Licciardi PV, Karagiannis TC: Dietary Sulforaphane in Cancer Chemoprevention: The Role of Epigenetic Regulation and HDAC Inhibition. Antioxid Redox Signal 2015;22:1382-1424. 